\title{
High expression of RAB43 predicts poor prognosis and is associated with epithelial-mesenchymal transition in gliomas
}

\author{
MING-ZHI HAN ${ }^{1,2}$, BIN HUANG ${ }^{1,2}$, AN-JING CHEN ${ }^{1,2}$, XIN ZHANG $^{1,2}$, \\ RAN XU ${ }^{1,2}$, JIAN WANG ${ }^{1-3}$ and XIN-GANG LI ${ }^{1,2}$ \\ ${ }^{1}$ Department of Neurosurgery, Qilu Hospital, Shandong University; ${ }^{2}$ Brain Science Research Institute, Shandong University, \\ Jinan, Shandong 250012, P.R. China; ${ }^{3}$ Department of Biomedicine, University of Bergen, 5009 Bergen, Norway
}

Received June 23, 2016; Accepted September 6, 2016

DOI: $10.3892 /$ or.2017.5349

\begin{abstract}
The Ras-related GTP-binding protein (RAB) family plays an important role in regulating signal transduction and cellular processes including vesicle transport, cytoskeleton formation and membrane trafficking. More recently, several $\mathrm{RAB}$ members have been reported to promote tumorigenesis in many types of cancers. However, the clinical significance and potential function of RAB43 in gliomas remain unclear. Herein, we found that RAB43 was upregulated and positively correlated with the grade of progression in glioma patients by in silico analysis and immunohistochemistry (IHC). Patients with high RAB43 displayed worse clinical outcomes in comparison to those with low RAB43. RAB43 was also highly expressed in mesenchymal and G3 subtypes, and isocitrate dehydrogenase 1 (IDH1) wild-type gliomas. Moreover, transcriptomic analyses via Gene Ontology (GO) and Kyoto Encyclopedia of Genes and Genomes (KEGG) pathways revealed that RAB43-related gene sets were mainly involved in the regulation of cell adhesion and cell migration processes. Further investigation indicated that RAB43 downregulation significantly suppressed the migratory and invasive ability of glioma cells, as well as decreased the expression of epithelial-mesenchymal transition (EMT) markers (N-cadherin, vimentin and Snail). In conclusion, a high level of RAB43 was significantly associated with the malignant phenotypes of gliomas, which suggests that RAB43 may serve as a novel biomarker and a potential therapeutic target for gliomas.
\end{abstract}

Correspondence to: Dr Jian Wang or Dr Xin-Gang Li, Department of Neurosurgery, Qilu Hospital, Shandong University, 107 Wen Hua Xi Road, Jinan, Shandong 250012, P.R. China

E-mail: jian.wang@biomed.uib.no

E-mail:1ixg@sdu.edu.cn

Key words: glioma, prognosis, RAB43, invasion, epithelial-mesenchymal transition

\section{Introduction}

Gliomas have been reported to be the most common type of primary intracranial tumors and account for $\sim 80 \%$ of all malignant brain tumors. According to the World Health Organization (WHO), gliomas are classified into 4 grades of malignancy (I-IV) based on their histological features, and glioblastoma multiform (GBM; grade IV) shows the worst prognosis, with a median survival time of only 12-15 months and a 5-year survival rate $<3 \%$ (1). In recent years, The Cancer Genome Atlas (TCGA) described a robust gene expression-based molecular classification of GBMs that divided them into 4 subtypes: proneural, neural, classical and mesenchymal, among which the mesenchymal subtype was distinguished from the others as being particularly malignant (2-5). Thus, identification of effective biomarkers for the mesenchymal type of glioma is eagerly awaited.

Ras-related GTP-binding protein 43 (RAB43) is a member of the Ras superfamily with a molecular weight of $23 \mathrm{kDa}$. Previous studies have shown that RAB43 is mainly located in endoplasmic reticulum and Golgi, and plays a role as a key regulator of vesicle movement, signal transduction and tethering membrane events in membrane trafficking (6). Recent studies have revealed that some of the RAB proteins are involved in the regulation of several signal transduction pathways relating to cell invasion, cell apoptosis and innate immune response $(7,8)$. Meanwhile, several members of RABs including RAB1B, RAB3D, RAB27A and RAB38 have been demonstrated to be dysregulated in a variety of malignant diseases and to play critical roles in tumor progression (9-12). Nonetheless, the differential expression, intracellular function and potential mechanism of RAB43 in tumors have not been reported.

In the present study, we compared the expression level of RAB43 in high-grade gliomas (HGGs) and low-grade gliomas (LGGs) and among different molecular subtypes based on the Chinese Glioma Genome (CGGA) and 4 additional independent microarray datasets. We also explored the relationship of RAB43 expression with clinicopathological parameters including overall survival (OS). Additionally, the protein expression level was validated in 52 glioma samples (WHO grade I-IV) by immunohistochemistry (IHC). Furthermore, we explored the potential biological impact of RAB43 on the invasive and metastatic properties of glioma 
cells, and the effects of RAB43 on cell migration and invasive capability were assessed in vitro.

\section{Materials and methods}

Clinical specimens and bioinformatics. The present study was approved by the Ethics Committee of Qilu Hospital. Archived paraffin-embedded glioma tissues were collected from 52 patients (WHO I-IV) who underwent surgery at the Department of Neurosurgery, Qilu Hospital of Shandong University (Shandong, China). Normal brain tissue samples $(n=5)$ were taken from trauma patients for whom partial resection of normal brain was required as decompression treatment for their severe head injuries. Written informed consent was obtained from all patients. Whole genome mRNA expression microarray data and clinical information from 310 samples [batch 1, 5 normal brain tissue (NBT) and 220 diffuse gliomas; batch 2, 85 diffuse gliomas] from CGGA (http://www.cgcg.org.cn/) were used for the analysis, containing 126 grade II, 51 grade III and 128 grade IV samples histologically diagnosed according to the WHO classification. Four external independent glioma databases (TCGA, Rembrandt, GSE16011 and GSE4290) were included as well.

$I H C$. Formalin-fixed, paraffin-embedded tissues of 52 specimens including different grades of astrocytic glioma were included. Endogenous HRP activity was blocked with 3\% $\mathrm{H}_{2} \mathrm{O}_{2}$. Antigen retrieval was achieved by boiling in sodium citrate buffer ( $\mathrm{pH}$ 6.0). After blocking with $10 \%$ normal goat serum, immunostaining was performed using a mouse anti-RAB43 monoclonal antibody (cat no. ab58030; Abcam, Cambridge, UK) at 1:50 dilution. Finally, the visualized signal was developed with 3,3'-diaminobenzidine (DAB) and the slides were counterstained in hematoxylin. The sections incubated with normal mouse serum instead of the primary antibody were used as negative controls. The results of the immunohistochemical staining were evaluated by two independent pathologists. The percentage of positive staining cells was scored as: $0-3$ ( 0 points for no cells stained, 1 points for $<25 \%, 2$ points for $25-75 \%$ and 3 points for $>75 \%$ of cells stained), and the intensity of immunoreactivity was also graded on a scale of $0-3$ scored as: $(0$, no staining; 1 , weak staining; 2 , moderate staining; and 3 , strong staining). The two scores were then multiplied to yield a total IHC score regarding the expression of RAB43 protein in a sample. Negative cases (-) had a total score of 0 , weakly positive $(+)$ cases had a total score of 1-2, moderately positive (++) cases had a score of 3-4, and strongly positive (+++) cases had a total score of 6-9.

Gene ontology (GO) and Kyoto Encyclopedia of Genes and Genomes (KEGG) analysis. Correlation analysis of RAB43 in whole genome gene expression profile was performed in the CGGA dataset $(n=305)$. To detect the biological processes and signaling pathways that correlate with RAB43 expression in glioma, RAB43 positively and negatively correlated genes $(\mathrm{p}<0.01)$ were analyzed by DAVID web tool (http://david.abcc. ncifcrf.gov/home. jsp).

Cell culture. U-87 MG and U-251 human GBM cell lines were purchased from the Culture Collection of the Chinese
Academy of Sciences (Shanghai, China), and cultured in Dulbecco's modified Eagle's medium (DMEM) supplemented with $10 \%$ fetal bovine serum (FBS) (both from Life Technologies, (Grand Island, NY, USA) and maintained at $37^{\circ} \mathrm{C}$ in an atmosphere of humidified air containing $5 \% \mathrm{CO}_{2}$.

RNA interference. Small interference RNA (siRNA) targeting RAB43 (5'-CCATTGAGACGTCTGCCAA-3') was synthesized by GenePharma Co., Ltd. (Shanghai, China). For transient silencing, $3 \times 10^{5}$ cells/well were seeded into 6-well plates and transfected with the relevant siRNA (100 nmol/well) using Lipofectamine RNAiMAX reagent (cat no. 13778150; Invitrogen, Carlsbad, CA, USA) following the manufacturer's protocol.

Western blotting. Total proteins were extracted in lysis buffer containing $50 \mathrm{mM}$ Tris- $\mathrm{HCl}, 150 \mathrm{mM} \mathrm{NaCl}, 1 \%$ sodium deoxycholate, $0.1 \%$ SDS, $20 \mathrm{mM}$ EDTA, $1 \mathrm{mM} \mathrm{NaF}$ and $1 \%$ Triton $\mathrm{X}-100(\mathrm{pH} 7.4)$ with protease inhibitors. The protein concentration was determined using the Bradford assay (Bio-Rad, Hercules, CA, USA). Lysis was run in a $10 \%$ sodium dodecyl sulfate-polyacrylamide electrophoresis (SDS-PAGE) gel, transferred to polyvinylidene difluoride (PVDF) membranes (Millipore, Billerica, MA, USA), incubated with antibodies against RAB43 (cat no. ab58030; Abcam), Snail (cat no. 3879), N-cadherin (cat no. 13116), vimentin (cat no. 12020), $\beta$-actin (cat no. 12262), MMP-2 (cat no. 13132) and MMP-9 (cat no. 13667) (all from Cell Signaling Technology, Beverly, MA, USA) diluted at 1:1,000.

Cell invasion and migration assays. For Transwell Matrigel invasion assays, $5 \times 10^{4}$ cells in $100 \mu \mathrm{l}$ of serum-free medium were plated onto the upper chamber of 24-well Transwell inserts ( $8-\mu \mathrm{m}$ pores; BD Biosciences, San Diego, CA, USA) coated with Matrigel. The lower chamber was filled with $600 \mu \mathrm{l}$ medium containing 20\% FBS. After 24-36 h, the non-invaded cells were gently scraped off by cotton swab. As for the migration assay, $2 \times 10^{4}$ cells in $100 \mu \mathrm{l}$ of serum-free medium were plated onto the upper chamber of the same inserts and migrated for $12 \mathrm{~h}$. The migrated cells were fixed with $10 \%$ formalin, and stained with crystal violet. Five random fields of each well were photographed and cell numbers were determined by Kodak Molecular Imaging (MI) software (Kodak, Rochester, NY, USA).

Statistical analysis. Survival curves were estimated by the Kaplan-Meier method and compared using the log-rank test. High or low expression was defined as higher or lower than the median value. Expression pattern of RAB43 in different subtypes and the associations of RAB43 with isocitrate dehydrogenase 1 (IDH1) mutation, methylation of $\mathrm{O}^{6}$-methylguanine-DNA methyltransferase (MGMT) promoter, co-deletion of $1 \mathrm{p} / 19 \mathrm{q}$, telomerase reverse transcriptase (TERT) loss, and alpha thalassemia/mental retardation syndrome X-linked (ATRX) mutation were performed in the CGGA and TCGA datasets. The one-way ANOVA test or t-test were used for all other data comparisons using GraphPad Prism 6 software. Pearson correlation was applied to evaluate the linear relationship between gene expression. A two-tailed $\chi^{2}$ test was used to determine the association between RAB43 
A
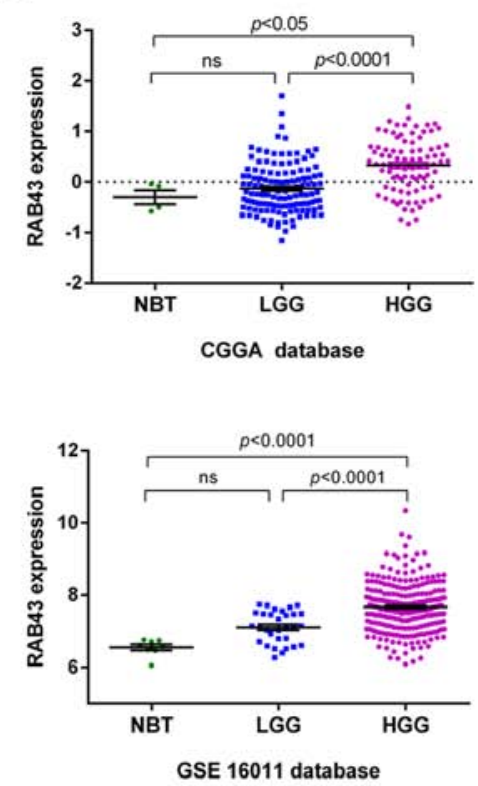
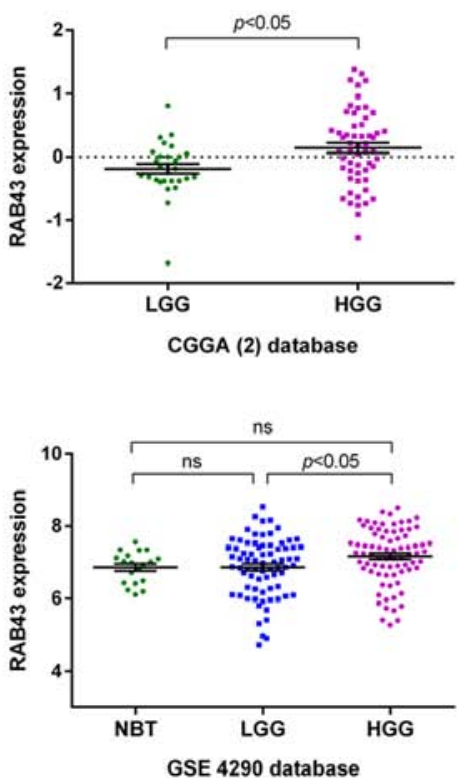
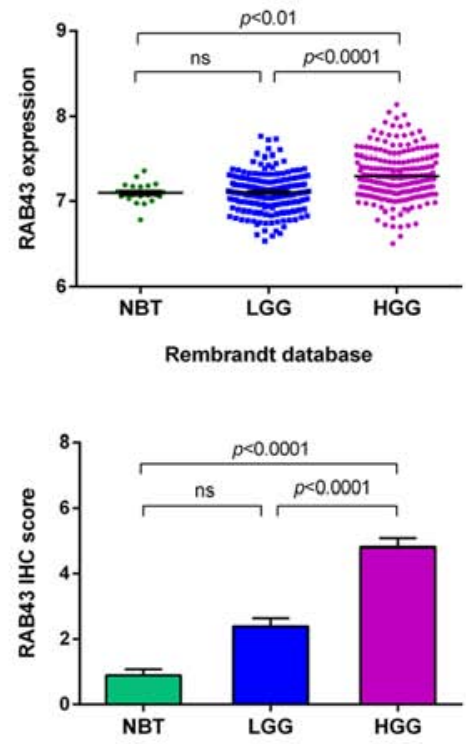

B
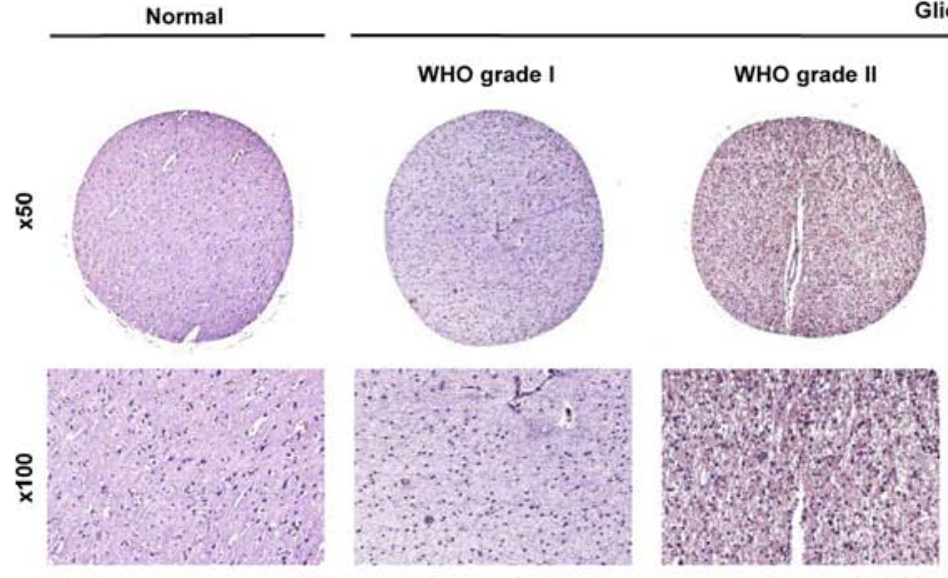

Gliomas
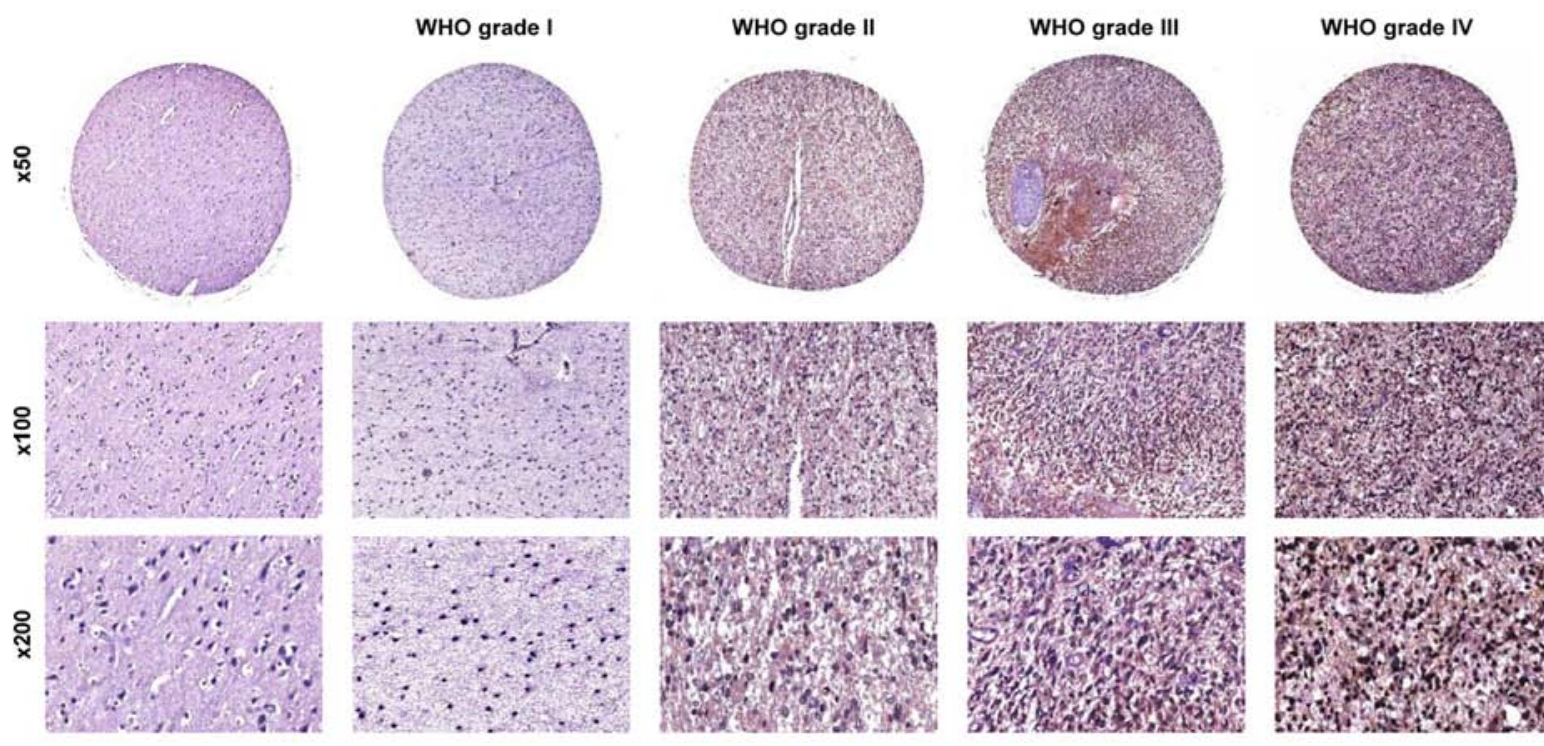

Figure 1. Differences in the expression of RAB43 in normal brain and glioma tissues. (A) The expression levels of RAB43 in glioma tissues were analyzed in CGGA, Rembrandt, GSE16011 and GSE4290 datasets as well as an independent cohort by IHC staining. A single spot represents the RAB43 expression value of an individual patient, with a line in the middle representing the median expression value and error bars representing the SEM. (B) Representative IHC staining images in human glioma of different WHO grades and normal brain specimens are shown as indicated. Magnification: x50, upper; x100, middle; x200, lower.

expression and clinicopathological characteristics. All data are presented as the mean \pm standard error. All tests were two-sided, and p-values $<0.05$ were considered to indicate a statistically significant result.

\section{Results}

Expression of RAB43 is associated with the grade of progression of the gliomas. By whole-genome expression profiling data, we compared the mRNA expression level of RAB43 in HGGs and LGGs as well as normal brain tissues (NBT). As shown in Fig. 1A, the results indicated that RAB43
mRNA expression level was significantly upregulated in the HGGs compared with that noted in the LGGs both in CGGA $(\mathrm{p}<0.0001)$ and 3 additional datasets. There were no significant differences between LGGs and NBT. Meanwhile, we analyzed the association of RAB43 with the clinicopathological characteristics of the glioma patients. As shown in Table I, the rate of high RAB43 expression tended to increase from grade II to IV according to the WHO classification ( $\mathrm{p}<0.0001)$. Furthermore, high expression of RAB43 was statistically associated with patients with age $\geq 45$ years $(p=0.0056)$. It was previously demonstrated that glioma patients with Karnofsky Performance Status (KPS) score $>80$ had a better prognosis 
A

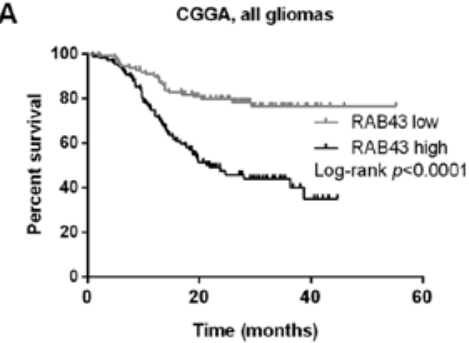

D

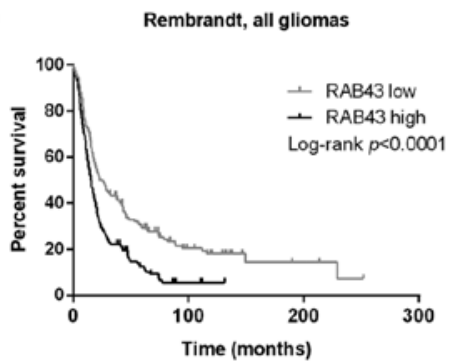

G

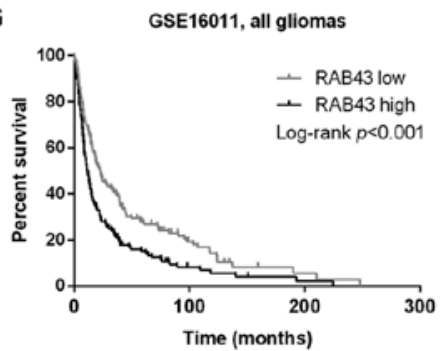

B

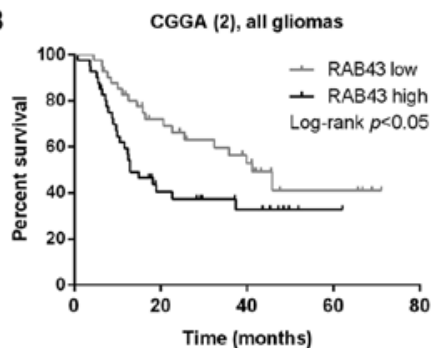

$E$

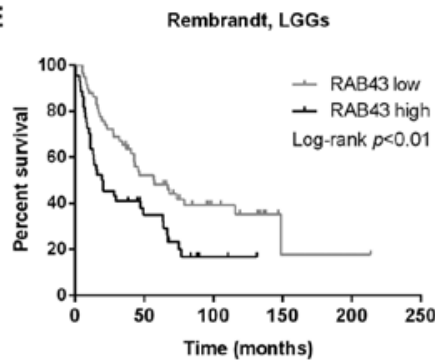

H

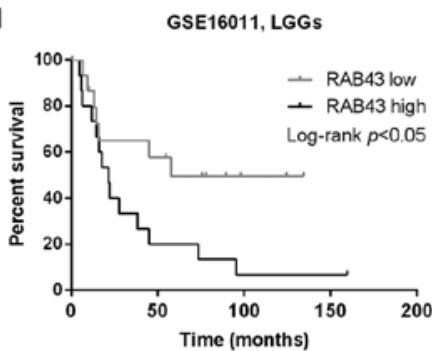

C

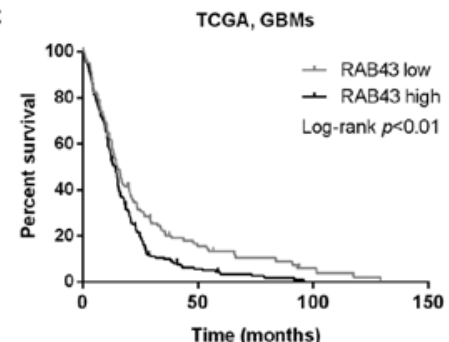

$\mathbf{F}$

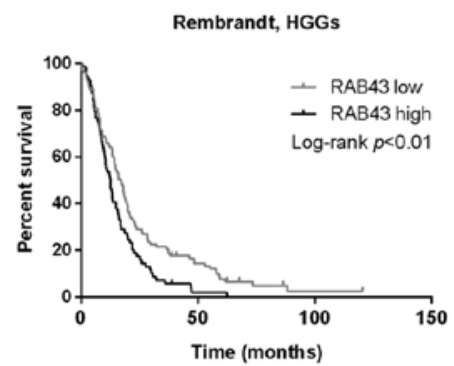

I

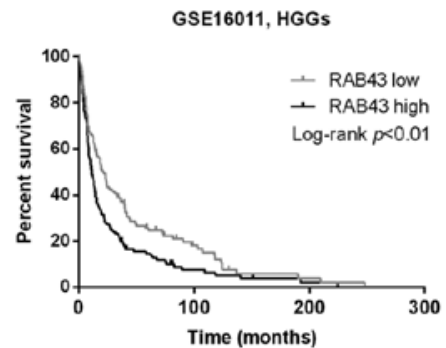

Figure 2. The prognostic value of RAB43 in gliomas. High RAB43 expression was related to shorter OS in (A and B) CGGA (batch 1, $\mathrm{n}=200$; batch 2, $\mathrm{n}=85$ ), (C) TCGA (n=348), (D-F) Rembrandt (n=329) and (G-I) GSE16011 (n=276) databases. High or low expression was defined as higher or lower than the median value. Kaplan-Meier plots were estimated according to different RAB43 gene expression for overall survival and p-values were obtained from log-rank test.

Table I. Clinical features of the glioma patients with differential expression of RAB43 in CGGA.

\begin{tabular}{lccr}
\hline Variable & $\begin{array}{c}\text { RAB43 } \\
\text { high expression } \\
(\mathrm{n}=152)\end{array}$ & $\begin{array}{c}\text { RAB43 } \\
\text { low expression } \\
(\mathrm{n}=153)\end{array}$ & P-value \\
\hline $\begin{array}{l}\text { Age (years }) \\
\geq 45\end{array}$ & 78 & 54 & $\mathbf{0 . 0 0 5 6}$ \\
$<45$ & 74 & 99 & \\
Gender & & & 0.4151 \\
Male & 94 & 87 & \\
Female & 58 & 66 & \\
KPS & & & 0.0675 \\
$\geq 80$ & 81 & 93 & \\
$<80$ & 29 & 17 & \\
WHO grade & & & $\mathbf{0 . 0 0 0 1}$ \\
II & 30 & 96 & \\
III & 32 & 19 & \\
IV & 90 & 39 & \\
\hline
\end{tabular}

P-values were determined by Chi-square and Fisher's exact tests. RAB, Ras-related GTP-binding protein 43; CGGA, Chinese Glioma Genome; KPS, Karnofsky Performance Status; WHO, World Health Organization. Bold print indicates a significant difference. than those with a KPS of $<80$ (13). However, no significant differences were identified between RAB43 expression in relation to KPS score $(\mathrm{p}=0.0675)$.

To further validate the expression pattern of RAB43, we detected the protein level of RAB43 in an independent group of 52 glioma patients and 5 NBT by IHC. RAB43 protein showed a higher expression status in the HGGs compared with the LGGs $(\mathrm{p}<0.0001)$, which was consistent with our findings in the mRNA microarrays (Fig. 1B). Thus, RAB43 was upregulated and positively correlated with the grade of progression both in silico and in glioma specimens.

High RAB43 expression is related to poor clinical outcomes in gliomas. The association of RAB43 expression with prognosis of the glioma patients was investigated through Kaplan-Meier survival curves. We found that patients with high RAB43 expression had a significant shorter OS time than those with low RAB43 expression (Fig. 2A and B). Similar results were found in the TCGA database among the 348 GBM patients (Fig. 2C). Moreover, the prognostic values of RAB43 in GSE16011 ( $\mathrm{n}=276)$ and Rembrandt databases $(\mathrm{n}=329)$ were analyzed, and the results indicated that high RAB43 was significantly associated with worse OS both in low- and high-grade glioma patients (Fig. 2D-I).

RAB43 expression shows subtype preference. Since RAB43 was upregulated in HGGs, we further screened its expression 
A

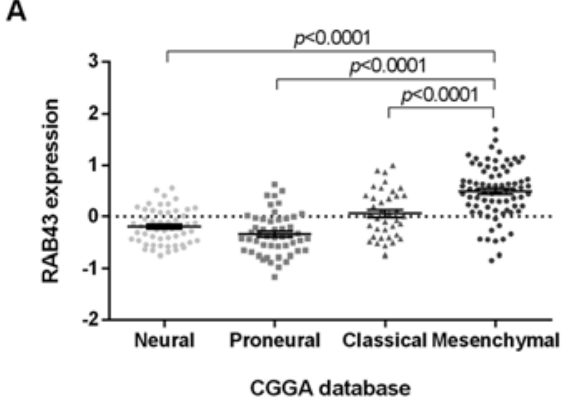

D

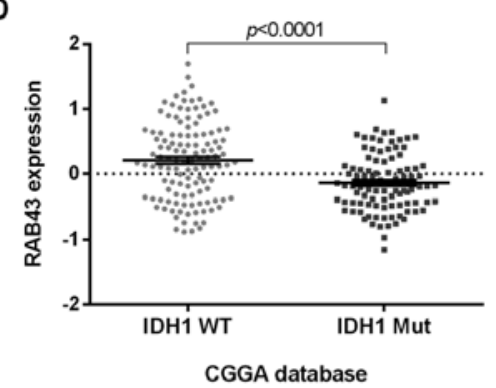

G

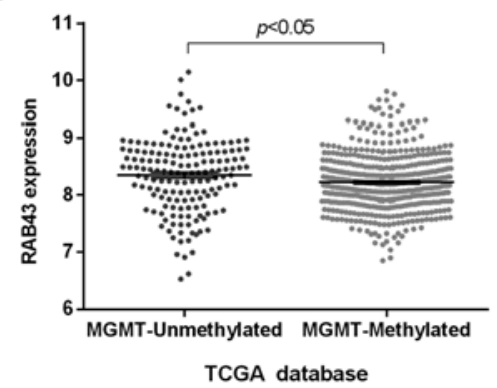

B

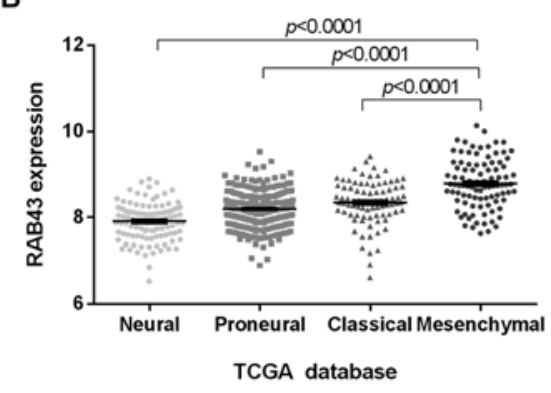

E

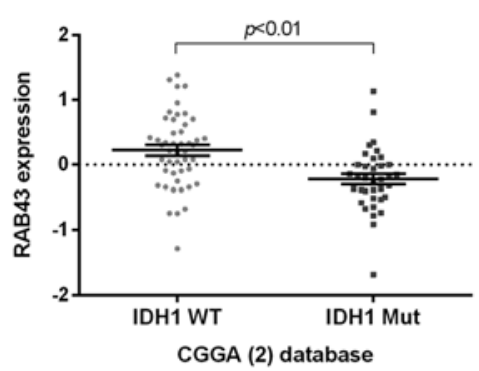

H

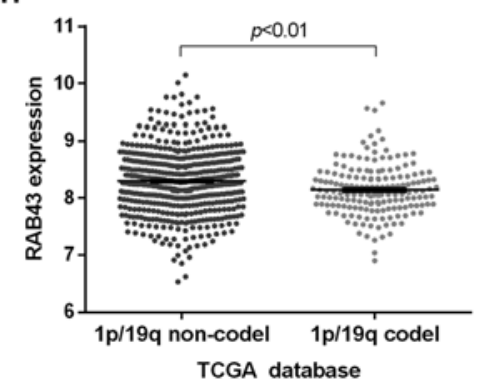

C

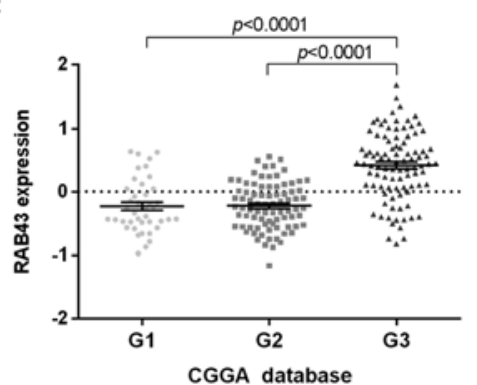

$\mathbf{F}$
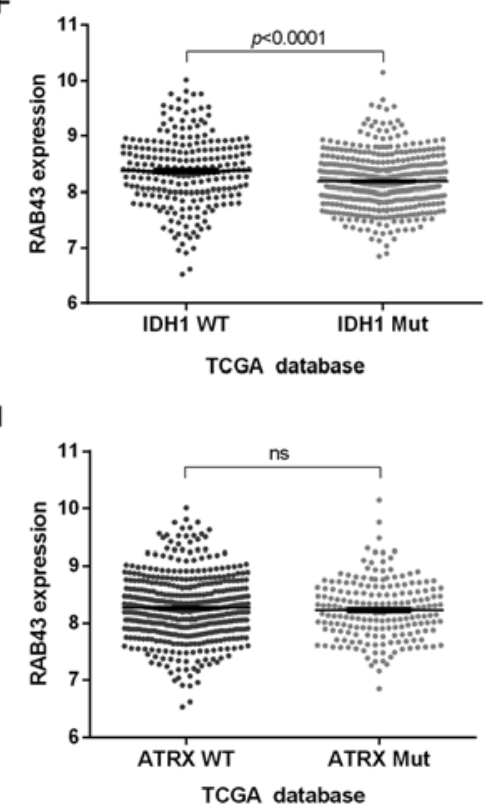

Figure 3. The expression features of RAB43 among TCGA and CGGA subtypes, and several genetic alterations. (A-C) A high RAB43 level was associated with mesenchymal and G3 subtypes, and patients with low RAB43 were more likely to be of the proneural subtype. (D-F) Patients with the wild-type IDH1 gene exhibited higher expression of RAB43 than those with mutant IDH1. (G-I) RAB43 was upregulated in glioma patients with unmethylated MGMT, 1p/19q non-co-deletion, but not with wild-type ATRX gene. A single spot represents the RAB43 expression value of an individual patient, with a line in the middle representing the median expression value and error bars representing the SEM.

pattern among different molecular subtypes. As shown in Fig. 3A-C, a high RAB43 level was associated with mesenchymal and G3 subtypes. In contrast, the patients with low RAB43 were more likely to be of the proneural subtype. More recently, some genetic alterations in glioma patients have been reported to predict favorable survival, including IDH1 mutation, methylation of MGMT, co-deletion of 1p/19q, TERT loss and ATRX mutation (14,15). We therefore analyzed whether RAB43 expression was correlated with these characteristics. As a result, patients with wild-type IDH1 gene showed higher expression of RAB43 than those with mutant IDH1 (Fig. 3D-F). Additionally, RAB43 was observed to be upregulated in glioma patients with unmethylated MGMT, $1 \mathrm{p} / 19 \mathrm{q}$ non-co-deletion, but not with the wild-type ATRX gene (Fig. 3G-I).

RAB43 is significantly associated with tumor cell adhesion and invasion. Next, to further understand the biological implications of RAB43 in gliomas, correlation analysis of RAB43 expression in whole genome gene profiling was performed in CGGA. As illustrated in the heatmap in Fig. 4, 1,165 genes were positively correlated and 382 genes were negatively correlated $(\mathrm{p}<0.01)$. Afterwards, GO analysis revealed that RAB43 was strongly associated with biological processes including response to stimulus, cell adhesion and migration, extracellular matrix organization, as well as response to wounding (Fig. 5A; Table II). In the KEGG analysis, the upregulated genes were enriched in pathways related to focal adhesion, cell adhesion molecules (CAMs) and ECM-receptor interaction in cancer progression (Fig. 5B). In contrast, the downregulated genes were enriched in developmental process, cell differentiation as well as neurogenesis (Fig. 5C and D).

Moreover, referring to the aberrant expression of RAB43 in HGGs and the mesenchymal subtype, co-expression of RAB43 with invasion or metastasis-related factors including matrix metalloproteinases (MMPs) was assessed. As shown in Fig. 6A-I, there was a significant positive correlation between RAB43, MMP-2 and MMP-9, as well as several mesenchymal subtype markers (vimentin, N-cadherin and Snail) $(\mathrm{p}<0.0001$, respectively). The RAB43 level was also found to be correlated with other key genes for malignant phenotypes in glioma, such as interleukin-6 (IL-6), IL-8, vascular endothelial growth factor A (VEGFA) and Ki-67. Overall, these functional 


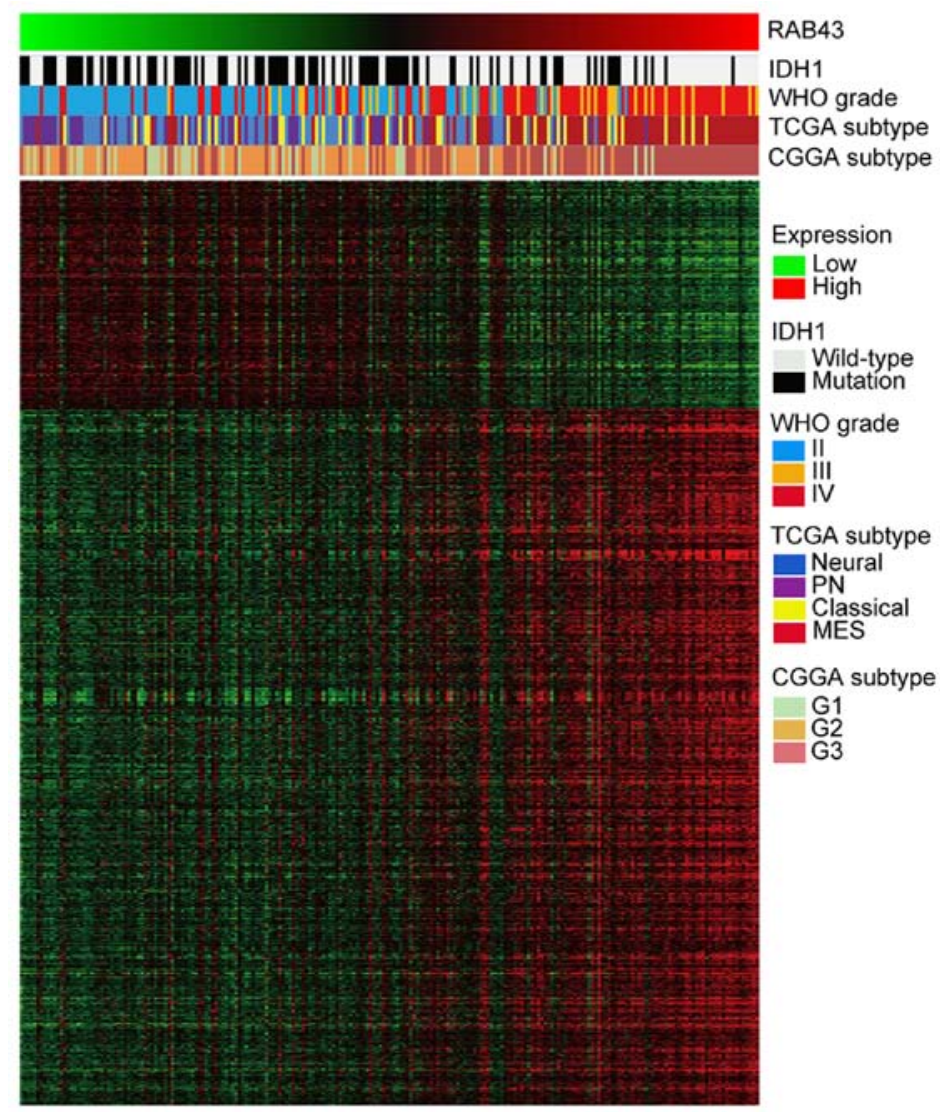

Figure 4. Analysis of RAB43-associated genes in whole genome gene expression profile. Based on the CGGA dataset, IDH1 status, WHO grade, TCGA as well as CGGA subtypes for each patient are listed in the upper part. The negatively and positively correlated genes for RAB43 are shown in the lower part. Columns represent patients and rows represent gene sets. Patients were arranged from left to right by increasing values of RAB43. Expression levels of the probe sets are represented by color, with green indicating lower and red indicating higher than the median value.

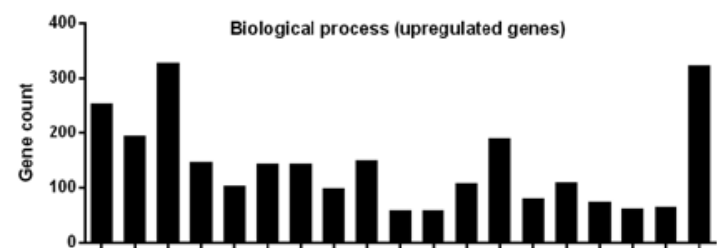

C
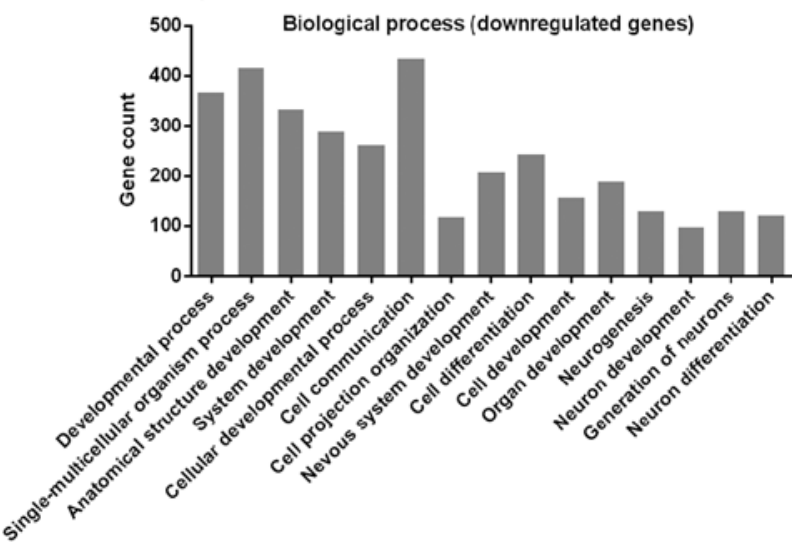
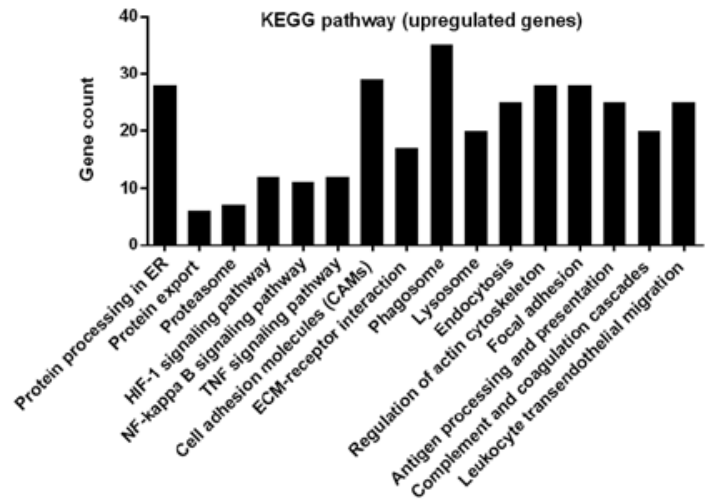

D

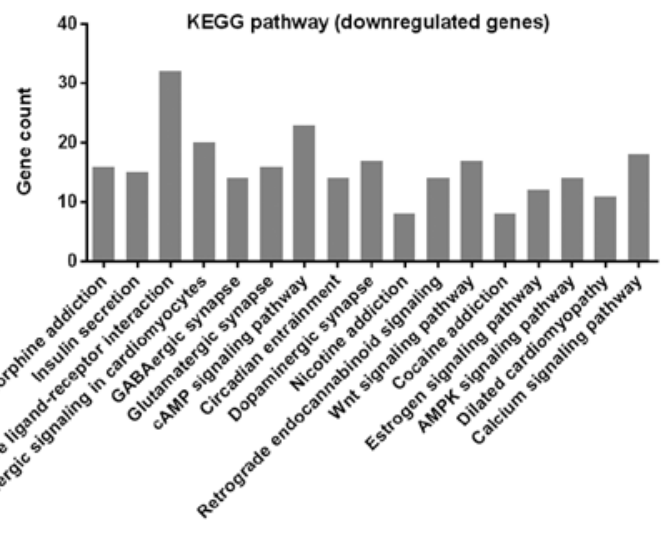

Figure 5. RAB43 is significantly associated with tumor cell adhesion and invasion. By Pearson correlation analysis, 1,165 genes were positively correlated and 382 genes were negatively correlated with RAB43 mRNA expression. Biological processes and KEGG pathway analysis of the (A and B) positively and (C and D) negatively correlated genes of RAB43 are shown. The potential functions and pathways are listed in the $\mathrm{x}$-axis. 
Table II. Gene sets enriched in the glioma samples with RAB43 high expression

\begin{tabular}{|c|c|c|c|}
\hline GO name & GO ID & Gene count & P-value \\
\hline Immune system process & GO:0002376 & 253 & $1.29 \mathrm{E}-76$ \\
\hline Response to external stimulus & GO:0009605 & 194 & $2.01 \mathrm{E}-48$ \\
\hline Positive regulation of biological process & GO:0048518 & 328 & $4.83 \mathrm{E}-48$ \\
\hline Locomotion & GO:0040011 & 147 & $1.29 \mathrm{E}-38$ \\
\hline Cell activation & GO:0001775 & 103 & $4.1 \mathrm{E}-37$ \\
\hline Cell adhesion & GO:0007155 & 144 & $6.11 E-37$ \\
\hline Biological adhesion & GO:0022610 & 144 & $8.46 \mathrm{E}-37$ \\
\hline Response to wounding & GO:0009611 & 99 & $2.74 \mathrm{E}-33$ \\
\hline Movement of cell or subcellular component & GO:0006928 & 150 & $9.24 \mathrm{E}-33$ \\
\hline Extracellular matrix organization & GO:0030198 & 60 & 4.19E-31 \\
\hline Extracellular structure organization & GO:0043062 & 60 & 4.78E-31 \\
\hline Cell migration & GO:0016477 & 108 & $8.08 \mathrm{E}-29$ \\
\hline Cell surface receptor signaling pathway & GO:0007166 & 190 & $1.25 \mathrm{E}-28$ \\
\hline Single organismal cell-cell adhesion & GO:0016337 & 80 & $3.18 \mathrm{E}-28$ \\
\hline Cell motility & GO:0048870 & 110 & $2.47 \mathrm{E}-27$ \\
\hline Wound healing & GO:0042060 & 74 & $2.5 \mathrm{E}-27$ \\
\hline Regulation of cytokine production & GO:0001817 & 63 & 4.84E-26 \\
\hline Regulation of cell adhesion & GO:0030155 & 66 & $2.2 \mathrm{E}-24$ \\
\hline Signal transduction & GO:0007165 & 324 & $4.47 \mathrm{E}-23$ \\
\hline
\end{tabular}

RAB, Ras-related GTP-binding protein 43; GO, Gene Ontology.

A

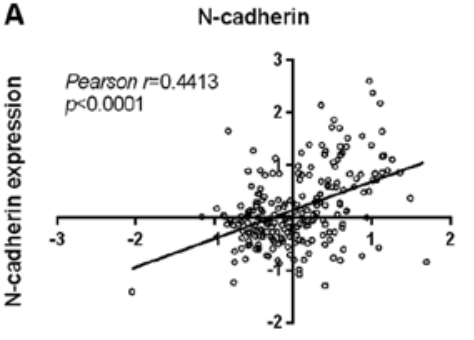

RAB43 expression

D

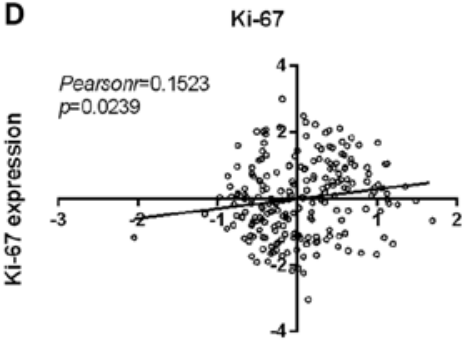

RAB43 expression

G

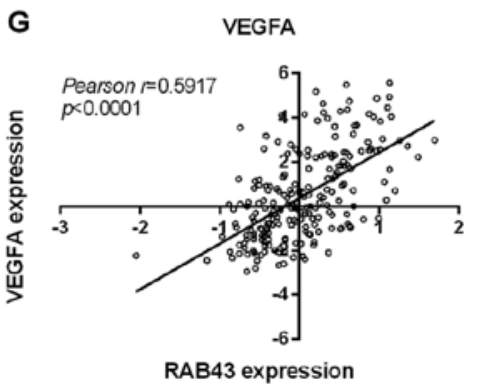

B

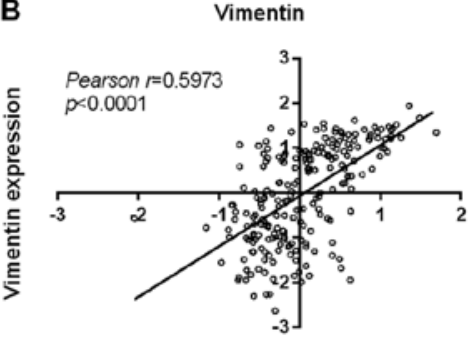

RAB43 expression

E

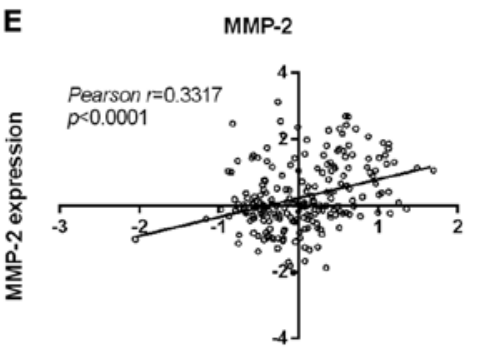

RAB43 expression

H

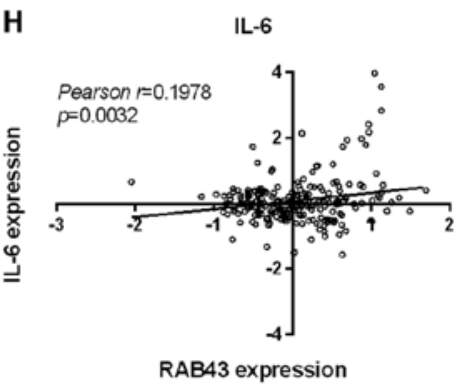

C

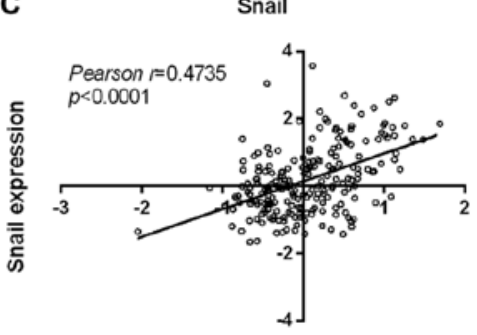

RAB43 expression

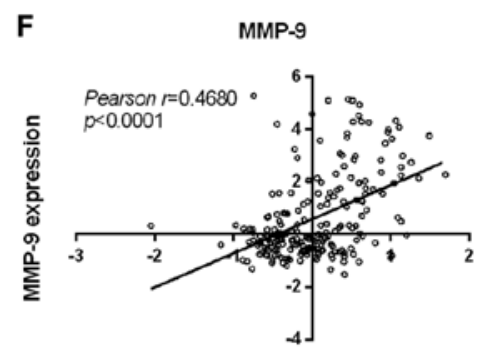

RAB43 expression

I

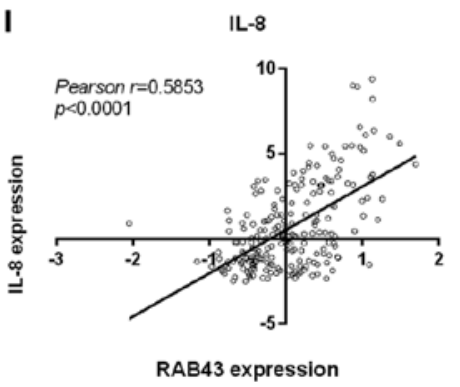

Figure 6. Correlations between RAB43 and several key genes in malignant gliomas. Correlations between RAB43 with (A) N-cadherin, (B) vimentin, (C) Snail, (D) Ki-67, (E) MMP-2, (F) MMP-9, (G) VEGFA, (H) IL-6 and (I) IL-8 levels in gliomas. The transcription levels of these genes were assessed and correlated using linear regression model. 
A

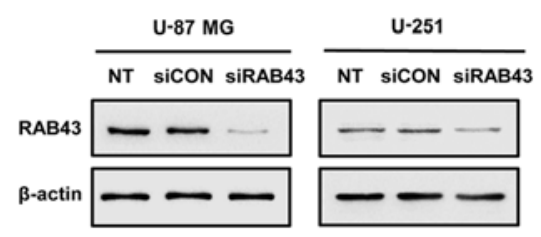

B

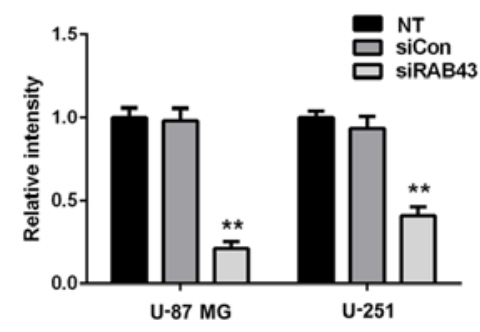

C

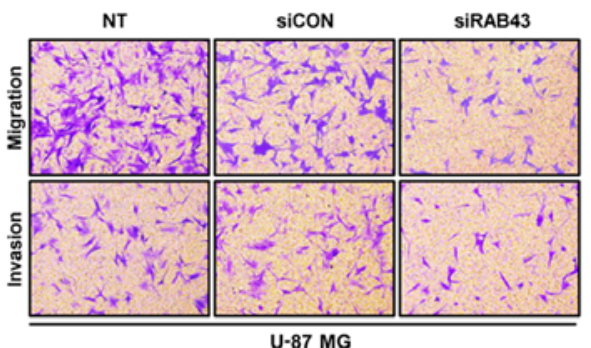

D

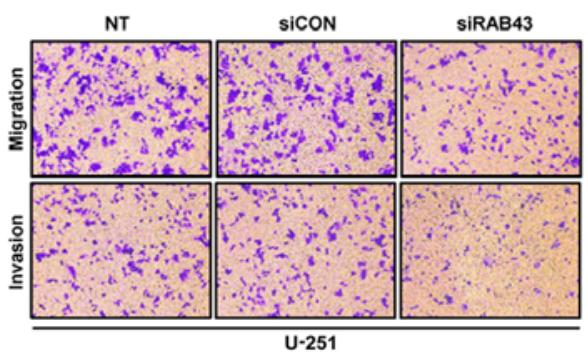

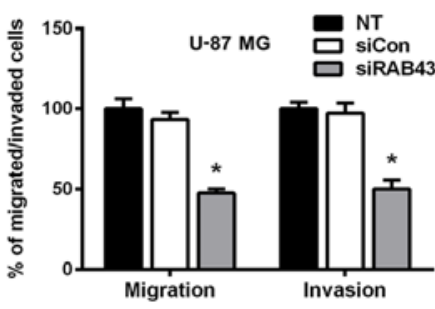

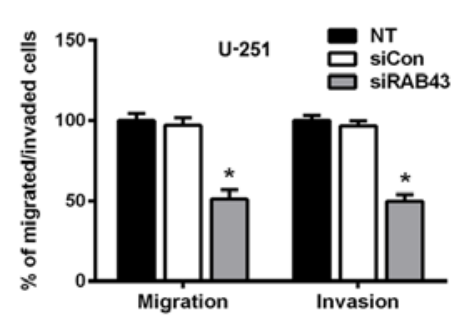

Figure 7. RAB43 knockdown affects the invasiveness and migration of glioma cell lines. (A and B) siRNA was designed to knock down the expression of RAB43, and downregulation of RAB43 in U-87 MG and U-251 human glioma cell lines were validated by western blotting. $\beta$-actin was used as a loading control. (C and D) Transfected cells were assayed for their migratory or invasive capability on Matrigel or Boyden chambers. Representative images of cells passing through the membrane are shown. Magnification, $x 100$. Data are shown as the mean \pm SEM from 3 independent experiments; ${ }^{*} p<0.05,{ }^{* *} p<0.01 . N T$, no treatment; siCON, non-silencing siRNA; siRAB43, siRNAs targeted against the RAB43 gene.

A

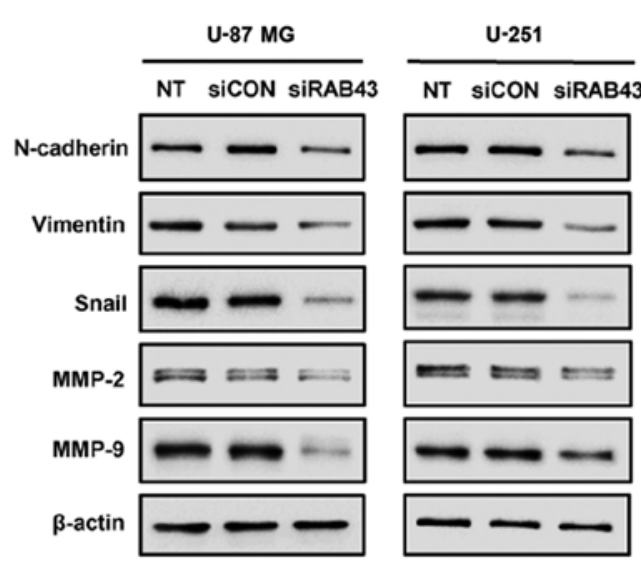

B

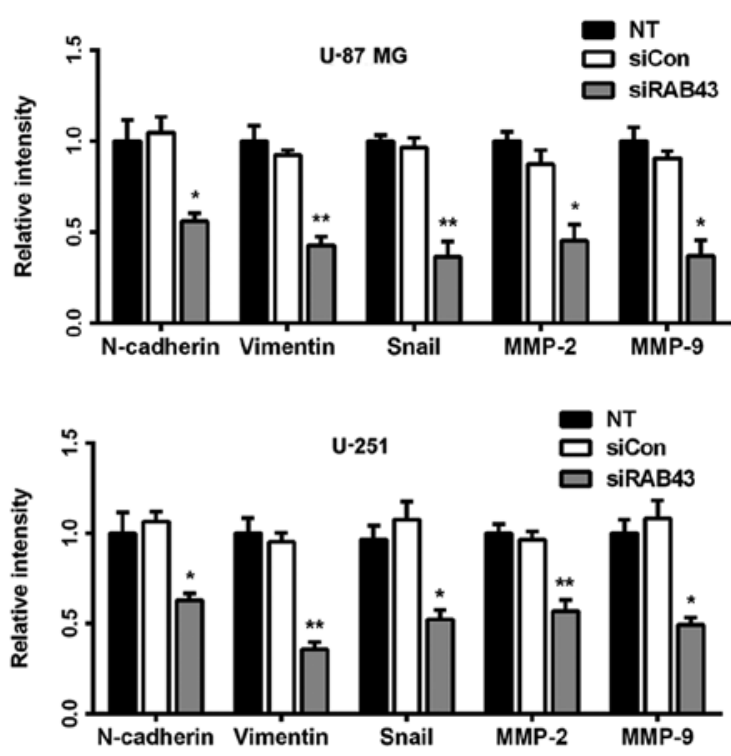

Figure 8. RAB43 contributes to the regulation of the EMT phenotype in glioma. (A and B) U-87 MG and U-251 cells were transfected as indicated. Expression profiles of EMT components in response to RAB43 knockdown as determined using western blotting. $\beta$-actin was used as a loading control. Data are shown as the mean \pm SEM from 3 independent experiments; ${ }^{*} \mathrm{p}<0.05,{ }^{* *} \mathrm{p}<0.01$. NT, no treatment; siCON, non-silencing siRNA; siRAB43, siRNAs targeted against the RAB43 gene.

analyses and correlation results implied the vital role of RAB43 as a potential oncogene in tumor migration and invasion.

RAB43 silencing reduces glioma cell migration and invasion. Next, we performed functional assays to determine the influence of RAB43 on glioma cell migration and invasion. By siRNA, RAB43 was transiently knockdown in U-87 MG (malignant glioma cell line annotated as mesenchymal subtype) and U-251 cells (Fig. 7A and B). Compared with the siCON group, silencing of RAB43 significantly inhibited both the migration and invasiveness of the U-87 MG glioma cells $(\mathrm{p}<0.05$; Fig. $7 \mathrm{C})$. Similar results were observed in the U-251 human glioma cells ( $p<0.05$; Fig. 7D). Meanwhile, downregulation of MMP-2 and MMP-9 levels was observed after silencing of RAB43 in the U-87 MG and U-251 cells (Fig. 8A). Taken together, these results indicated that suppression of RAB43 significantly inhibited the migration and invasion of glioma cells. 
RAB43 knockdown inhibits mesenchymal properties in glioma cells. Enhanced cell migration and invasion capabilities are important consequences of epithelial-mesenchymal transition (EMT), an early event in tumor metastasis (16). Therefore, we examined the expression of molecules associated with EMT in glioma cells. As shown in Fig. 8A and B, RAB43 knockdown significantly suppressed expression of mesenchymal markers (N-cadherin and vimentin), as well as EMT-related transcription factor Snail in both the U-87 MG and U-251 cells. Thus, RAB43 expression may contribute to the invasiveness and poor prognosis in patients by promoting EMT in glioma cells.

\section{Discussion}

Recently, several members of the RAB family have been indicated to participate in cancer progression (17). For instance, RAB25 contributes to tumor progression by directing the localization of integrin-recycling vesicles and promoting the invasive ability of tumor cells in breast and ovarian cancers $(18,19)$. RAB3D was reported to play a crucial role in invasion and metastasis via the EMT process in colorectal cancer (10). As a member of the RAB family, RAB43 was identified to be located in endoplasmic reticulum and Golgi, which plays crucial roles in protein transport and Golgi organization (6). However, the effect of RAB43 in tumors has not been previously reported. In the present study, according to the mRNA microarray of CGGA, RAB43 was highly expressed in HGGs in comparison with LGGs (Fig. 1A). Due to differences in the genetic background between populations, we subsequently validated these findings in 4 published cohorts. In addition, the protein level of RAB43 was investigated by IHC from an independent group of patients ( $n=52$; Fig. 1B), and similar findings were observed. Moreover, patients with high RAB43 had worse overall survival than those with low RAB43 in both LGGs and HGGs (Fig. 2). Collectively, these data suggest that RAB43 may be associated with the malignant phenotypes of gliomas and could serve as a novel prognostic indicator in clinical practice.

Characterized by particular molecular signatures, glioma has recently been classified into distinct molecular subtypes, among which the mesenchymal subtype-associated genes were more related to aggressive behavior in tumors and conferred a poor prognosis in patients (20). Recently, CGGA derived 3 subgroups of gliomas including G1, G2 and G3 with differences in clinical characteristics based on the Chinese population (5). The G1 subgroup was characterized by favorable clinical outcome, young age, low malignant behaviors and high IDH1 mutation, while G3 groups exhibited contrasting characteristics with poor prognosis and low rate of IDH1 mutation. Notably, consistent with the worse outcomes of patients with high RAB43, we found a significantly increased expression of RAB43 in mesenchymal and G3 subtypes, and IDH1 wild-type patients. In contrast, patients with low RAB43 were more likely to be of the proneural subtype and IDH1 mutation patients. Additionally, RAB43 was also highly expressed in glioma patients with unmethylated MGMT and 1p/19q non-co-deletion (Fig. 3).

To further explore the biological relevance of the RAB43 transcriptome, functional clustering annotation and integration into KEGG and GO analysis were performed based on the
CGGA dataset. GO analyses revealed that RAB43-assosiated genes showed significant enrichment mainly in biological processes related to cell adhesion and migration, extracellular matrix organization, as well as response to wounding (Fig. 5A; Table II). In the KEGG analysis, the upregulated genes were enriched in pathways related to focal adhesion, cell adhesion molecules (CAMs), ECM-receptor interaction in cancer progression and immune-related pathways (Fig. 5B). Together, these data further implicate the critical role of RAB43 in invasiveness and metastasis of gliomas.

Excessive migration and invasion are hallmarks of malignant tumors. Recent studies suggest a role of matrix metalloproteinases (MMPs) in the process of glioma cell invasion (21,22). Among all MMPs, MMP-2 and MMP-9 play important roles in basement membrane type IV collagen degradation during tumor migration and invasion (23). In the present study, we evaluated the influence of RAB43 alteration on the migration and invasion of glioma cells. As expected, RAB43 knockdown prominently reduced the migratory and invasive abilities of the glioma cells (Fig. 7C and D). Meanwhile, MMP-2 and MMP-9 were also significantly reduced after RAB43 knockdown (Fig. 8), which suggest the role of RAB43 in glioma metastasis.

It has been reported that EMT occurs during tumor progression, leading to increased motility and invasiveness of cancer cells. An obvious characteristic of EMT is upregulation of mesenchymal markers such as $\mathrm{N}$-cadherin, vimentin and Snail (24-26). However, the impact of the RAB family on EMT processes remain unclear. Hence, we evaluated whether RAB43 was involved in the regulation of EMT-specific proteins. As shown in Fig. 8A and B, the siRAB43 group showed significant decreases in $\mathrm{N}$-cadherin, vimentin and Snail expression. Taken together, these findings imply that RAB43 expression may contribute to metastasis and poor prognosis in patients by promoting EMT in glioma cells. However, further investigation is still needed to elucidate the regulatory mechanisms of RAB43 in the EMT process.

In summary, the present study demonstrated for the first time that RAB43 is overexpressed in glioma tissues, and increased expression of RAB43 is associated with poor prognostic features. In addition, high RAB43 expression is related to the mesenchymal and G3 subtypes, as well as the wild-type IDH1 gene in glioma patients. In vitro experiments revealed that RAB43 regulates EMT and the invasiveness of glioma cells. Altogether, RAB43 may serve as a novel biomarker and a potential therapeutic target for malignant gliomas.

\section{Acknowledgements}

The present study was supported by grants from the Natural Science Foundation of China (nos. 81502164, 81402060 and 81572487), the Shandong Provincial Natural Science Foundation (BS2015 YY004 and BS2014YY033), the Special Foundation for Taishan Scholars (nos. ts20110814 and tshw201502056), the Fundamental Research Funds of Shandong University, the Department of Science and Technology of Shandong Province (2015GGE27101 and 2015ZDXX0801A01), the University of Bergen, The Helse Bergen, Norway and the Norwegian Centre for International Cooperation in Education (SIU) (UTF-2014/10047). 


\section{References}

1. Wen PY and Kesari S: Malignant gliomas in adults. N Engl J Med 359: 492-507, 2008.

2. Brennan C: Genomic profiles of glioma. Curr Neurol Neurosci Rep 11: 291-297, 2011.

3. Huse JT, Phillips HS and Brennan CW: Molecular subclassification of diffuse gliomas: Seeing order in the chaos. Glia 59: 1190-1199, 2011

4. Sulman EP and Aldape K: The use of global profiling in biomarker development for gliomas. Brain Pathol 21: 88-95, 2011.

5. Yan W, Zhang W, You G, Zhang J, Han L, Bao Z, Wang Y, Liu Y, Jiang C, Kang C, et al: Molecular classification of gliomas based on whole genome gene expression: A systematic report of 225 samples from the Chinese Glioma Cooperative Group. Neuro Oncol 14: 1432-1440, 2012.

6. Dejgaard SY, Murshid A, Erman A, Kizilay O, Verbich D, Lodge R, Dejgaard K, Ly-Hartig TB, Pepperkok R, Simpson JC, et al: Rab18 and Rab43 have key roles in ER-Golgi trafficking. J Cell Sci 121: 2768-2781, 2008.

7. Lu N and Zhou Z: Membrane trafficking and phagosome maturation during the clearance of apoptotic cells. Int Rev Cell Mol Biol 293: 269-309, 2012.

8. Wang R, Zhang Y, Liu S, Li C, Sun L, Bao L, Feng J and Liu Z: Analysis of $52 \mathrm{Rab}$ GTPases from channel catfish and their involvement in immune responses after bacterial infections. Dev Comp Immunol 45: 21-34, 2014.

9. Nikoshkov A, Broliden K, Attarha S, Sviatoha V, Hellström AC, Mints $M$ and Andersson S: Expression pattern of the PRDX2, RAB1A, RAB1B, RAB5A and RAB25 genes in normal and cancer cervical tissues. Int J Oncol 46: 107-112, 2015.

10. Luo Y, Ye GY, Qin SL, Mu YF, Zhang L, Qi Y, Qiu YE, Yu MH and Zhong M: High expression of Rab3D predicts poor prognosis and associates with tumor progression in colorectal cancer. Int J Biochem Cell Biol 75: 53-62, 2016.

11. Wang H, Zhao Y, Zhang C, Li M, Jiang C and Li Y: Rab27a was identified as a prognostic biomaker by mRNA profiling, correlated with malignant progression and subtype preference in gliomas. PLoS One 9: e89782, 2014.

12. Wang $\mathrm{H}$ and Jiang C: RAB38 confers a poor prognosis, associated with malignant progression and subtype preference in glioma. Oncol Rep 30: 2350-2356, 2013.

13. Tortosa A, Viñolas N, Villà S, Verger E, Gil JM, Brell M, Caral L, Pujol T, Acebes JJ, Ribalta T, et al: Prognostic implication of clinical, radiologic, and pathologic features in patients with anaplastic gliomas. Cancer 97: 1063-1071, 2003.

14. Jiang T, Mao Y, Ma W, Mao Q, You Y, Yang X, Jiang C, Kang C, Li X, Chen L, et al; Chinese Glioma Cooperative Group (CGCG): CGCG clinical practice guidelines for the management of adult diffuse gliomas. Cancer Lett 375: 263-273, 2016.
15. Louis DN, Perry A, Reifenberger G, von Deimling A, FigarellaBranger D, Cavenee WK, Ohgaki H, Wiestler OD, Kleihues P and Ellison DW: The 2016 World Health Organization Classification of Tumors of the Central Nervous System: A summary. Acta Neuropathol 131: 803-820, 2016.

16. Kahlert UD, Nikkhah G and Maciaczyk J: Epithelial-tomesenchymal(-like) transition as a relevant molecular event in malignant gliomas. Cancer Lett 331: 131-138, 2013.

17. Subramani D and Alahari SK: Integrin-mediated function of Rab GTPases in cancer progression. Mol Cancer 9: 312, 2010.

18. Caswell PT, Spence HJ, Parsons M, White DP, Clark K, Cheng KW, Mills GB, Humphries MJ, Messent AJ, Anderson KI, et al: Rab25 associates with alpha5betal integrin to promote invasive migration in 3D microenvironments. Dev Cell 13: 496-510, 2007.

19. Cheng KW, Lahad JP, Kuo WL, Lapuk A, Yamada K, Auersperg N, Liu J, Smith-McCune K, Lu KH, Fishman D, et al: The RAB25 small GTPase determines aggressiveness of ovarian and breast cancers. Nat Med 10: 1251-1256, 2004.

20. Arimappamagan A, Somasundaram K, Thennarasu K, Peddagangannagari S, Srinivasan H, Shailaja BC, Samuel C, Patric IR, Shukla S, Thota B, et al: A fourteen gene GBM prognostic signature identifies association of immune response pathway and mesenchymal subtype with high risk group. PLoS One 8: e62042, 2013.

21. Liu L, Wu J, Ying Z, Chen B, Han A, Liang Y, Song L, Yuan J, $\mathrm{Li} \mathrm{J}$ and $\mathrm{Li} \mathrm{M}$ : Astrocyte elevated gene-1 upregulates matrix metalloproteinase- 9 and induces human glioma invasion. Cancer Res 70: 3750-3759, 2010.

22. Egeblad M and Werb Z: New functions for the matrix metalloproteinases in cancer progression. Nat Rev Cancer 2: 161-174, 2002.

23. Tester AM, Ruangpanit N, Anderson RL and Thompson EW: MMP-9 secretion and MMP-2 activation distinguish invasive and metastatic sublines of a mouse mammary carcinoma system showing epithelial-mesenchymal transition traits. Clin Exp Metastasis 18: 553-560, 2000.

24. Cano A, Pérez-Moreno MA, Rodrigo I, Locascio A, Blanco MJ, del Barrio MG, Portillo F and Nieto MA: The transcription factor snail controls epithelial-mesenchymal transitions by repressing E-cadherin expression. Nat Cell Biol 2: 76-83, 2000.

25. Yan LX, Liu YH, Xiang JW, Wu QN, Xu LB, Luo XL, Zhu XL, Liu C, Xu FP, Luo DL, et al: PIK3R1 targeting by miR-21 suppresses tumor cell migration and invasion by reducing PI3K/AKT signaling and reversing EMT, and predicts clinical outcome of breast cancer. Int J Oncol 48: 471-484, 2016.

26. Yokoyama K, Kamata N, Fujimoto R, Tsutsumi S, Tomonari M, Taki M, Hosokawa $\mathrm{H}$ and Nagayama $\mathrm{M}$ : Increased invasion and matrix metalloproteinase- 2 expression by Snail-induced mesenchymal transition in squamous cell carcinomas. Int J Oncol 22: 891-898, 2003. 